\title{
Richard Wilhelm als Kulturvermittler und sein Einfluss auf Hermann Hesse
}

\section{Chen Zhuangying}

In der Geschichte der menschlichen Zivilisation hat sich die Definition von Helden aufgrund von Geographie, Ethik, Kultur und sozialer Entwicklungsstufe ständig geändert. Die Helden der antiken griechischen und römischen Mythologie unterscheiden sich stark von denjenigen der chinesischen. Zugleich vermitteln diese Heldenbilder auch unterschiedliche Werte und Normen, verkörpern andere Vorbilder. ${ }^{1}$ In der griechischen Mythologie sowie im Christen- und Judentum ist der Mensch Gottes Schöpfung und was der Mensch hat, ist letztlich Gottes Geschenk. Der Mensch muss den Anordnungen der höheren Macht folgen, andernfalls wird er bestraft. So wird Sisyphos zu ewiger Qual verurteilt, weil er die Götter überlisten, Prometheus, weil er gegen ihren Willen den Menschen helfen wollte; Adam und Eva werden aus dem Paradies verbannt, weil sie Gottes Gebot nicht Folge leisteten. Kurz gesagt, im westlichen religiösen Denken ist der Mensch Gott bzw. den Göttern unterworfen und weist inhärente Mängel auf, die ihren prägnantesten Ausdruck in der Vorstellung von der Erbsünde finden. Gott und Himmel hingegen sind fehlerfrei oder, wenn auch nicht völlig erhaben über menschliche Schwächen und Laster, so doch zumindest stärker, mächtiger und verständiger, in jedem Falle Gehorsam gebietend; es sind die Götter im Himmel, die die Menschheit führen. Als Gott all das Leid und Übel auf der von ihm geschaffenen Erde sah, löschte er die Menschheit kurzerhand durch die Sintflut aus. Er hat jederzeit die Macht, seine eigene Schöpfung wieder zurückzunehmen. Die Arche Noah symbolisiert hierbei nicht nur die Hoffnung, sondern in erster Linie die Schwäche und Machtlosigkeit des Menschen, der einer höheren Macht hilflos ausgeliefert ist. Der Mensch der Bibel ist ein unvollkommener, mit Fehlern und Lastern behafteter Sünder, winzig klein und unvermögend im Vergleich zum Göttlichen.

In der chinesischen Mythologie hingegen herrscht ein ganz anderes Menschenbild vor: Er wird als gottesebenbürtig, nie dem Schicksal unterworfen und ständig kampfbereit beschrieben. Sein Schicksal liegt in seinen eigenen Händen, von Geburt her ist er bestimmt, die Natur zu bändigen und sich für das Wohl und Interesse der Familie, der Gesellschaft und des Vaterlandes zu opfern. Dieser Kampfgeist und Opfersinn für ein höheres Ziel wird in der chinesischen Kultur besonders großgeschrieben, was sich in vielen traditionellen Erzählungen widerspiegelt. Eine berichtet von einem Mädchen, das im Meer ertrank und das, nachdem sie als Vogel wiedergeboren worden war, immer wieder mit Steinen im Schnabel über den Oze-

1 Vgl. Ulrich Unger: Heroen, Helden und Eisenfresser. Zur Anthropologie der Tapferkeit, in: Hans Stumpfeldt / Martin Hanke (Hg.): Kleine Schriften, Gossenberg 2009, S. 225-238. 
an flog, die sie über den Wellen abwarf, um das Meer damit aufzufüllen. Eine andere erzählt von einem großen Berg, der vor der Haustür eines alten Mannes stand. Statt einen Umweg zu finden, versuchte er den Berg wegzuschaufeln. Während Noah in seiner Ohnmacht kaum sich und seine Familie vor der Sintflut zu retten vermochte, trommelte der chinesische König Yu sein Volk zusammen und trug ihm auf, Gräben auszuheben, um die Sintflut ins Meer zu abzuleiten. Und auch dem anderen Element war man nicht hilflos ausgeliefert: Einst standen zehn Sonnen am Himmel und drohten durch ihre Hitze die Erde zu verbrennen. Als die Menschen das nicht länger ertrugen, leisteten sie Widerstand und der Held Hou Yi schoss mit seinem Bogen neun Sonnen vom Himmel herunter. Die letzte Sonne, zitternd am Himmel stehend, wurde durch diese Heldentat in ihre Schranken gewiesen und diente fortan gehorsam den Menschen.

Auch wenn die grundsätzliche Bejahung der Natur ein wesentliches Element der chinesischen Kultur darstellt, so kennt sie nicht weniger die Bändigung der Natur und den Kampf gegen sie. So lautet ein Grundsatz des Philosophen Lao Tse: „Die Welt ist nicht gütig, alles ist ein Hund, der Heilige ist nicht gütig und die Menschen sind Hunde. ${ }^{\text {" } 2}$ Was Lao Tse mit diesem Spruch meint, ist eindeutig: Auf der Welt ist man auf sich alleine gestellt. Um zu überleben, muss man kämpfen, kann sich weder auf Götter noch Heilige verlassen. Daher ist in den beiden großen Philosophien Chinas, dem Taoismus und Konfuzianismus, keine Spur eines Gottes zu finden. In einem ähnlichen Sinne schrieb 1917 Mao Zedong, angeregt von der Russischen Revolution: „Es ist eine unendliche Freude, den Himmel zu bekämpfen. Es ist eine unendliche Freude, die Erde zu bekämpfen. Es ist ebenfalls eine unendliche Freude, die Menschen zu bekämpfen.. ${ }^{3}$

In der chinesischen Kultur nimmt also der Mensch in der Definition des Heroischen eine zentrale Rolle ein. Diejenigen, die sich mit edler Moral, Tapferkeit und Selbstlosigkeit für Volk und Land opfern, sich vorbildhaft für das Gemeinwohl einsetzen, werden von der Öffentlichkeit bewundert, respektiert und als Helden anerkannt. Dazu nun gehören auch einige Missionare, denen man wegen ihres Beitrags zur Kulturvermittlung und Völkerverständigung zwischen China und dem Abendland heroische Qualitäten zusprach.

Bereits seit der Frühen Neuzeit waren Missionare aus dem Westen in China aktiv. Zumeist aber wurden sie von chinesischer Seite weniger für ihre christliche Missionsarbeit geehrt als vielmehr für ihre Leistungen bei der Vermittlung von Bildung, Wissenschaft, Technologie, Medizin und Astronomie. Das durch sie vermittelte Wissen steigerte vielfach die Lebensqualität und das Bildungsniveau vieler Menschen und trieb ganz grundsätzlich die gesellschaftliche Aufklärung voran. Diese Missionare sind in den Augen zahlreicher Chinesen zu Helden geworden.

Ein frühes Beispiel ist der italienische Missionar Matteo Ricci (1552-1610), ein Pionier des Katholizismus in China, der die moderne Mathematik, Geographie

2 Lao Tse: Tao Te King, Xian 1995, S. 17.

3 Mao Zedong: Biographie von Mao Zedong, Bd. 1, Peking 2013, S. 27. 
und Geometrie nach China brachte. Er veröffentlichte als Erster eine Weltkarte und die chinesische Version der Euklidischen Geometrie in China, übersetzte darüber hinaus mehr als 150 wissenschaftliche Werke aus dem Westen ins Chinesische.

Der deutsche Missionar Johann Adam Schall von Bell (1592-1666) schaffte es gar, Lehrer des Kaisers Kang Xi und hochrangiger Hofbeamter zu werden. Er verbesserte den chinesischen Mondkalender, um ihn für die landwirtschaftliche Aussaat und den astrologischen Gebrauch geeigneter zu machen, führte die moderne Artillerie-Produktionstechnologie ein und wurde noch nach dem Tod mit hohen Ehren versehen.

Sowohl Ricci als auch von Bell wurden von den Chinesen als Helden der Kulturvermittlung verehrt. Während die beiden Missionare Wissenschaft und Technik aus dem Westen nach China brachten, machte sich einige Jahrhunderte später ein anderer Missionar in China einen großen Namen, der in genau der entgegengesetzten Richtung wirkte und entscheidend dazu beitrug, dass China mit seiner fünftausend Jahre alten Kultur und Philosophie im Westen bekannt, studiert, bewundert und schließlich als der griechischen Antike ebenbürtig anerkannt wurde. Er war nicht nur ein Missionar, sondern auch ein erfolgreicher Pädagoge und renommierter Sinologe, in den Augen der Chinesen ein Held der Kulturvermittlung zwischen Ost und West, gleichsam ein Deutsch-Chinese: Richard Wilhelm, auf Chinesisch: Wei Lixian.

Darf ein gescheiterter Missionar, der zur Verbreitung des Christentums in China kaum etwas beigetragen hat, als Held bezeichnet werden? Aus christlicheuropäischer Perspektive scheint das zweifelhaft, nach chinesischen Maßstäben allerdings weist er durchaus heroische Züge auf. Er mag Deutscher gewesen sein, doch verbrachte er immerhin über 25 Jahre seines Lebens in China. Dort gewann er in den Kreisen der Intellektuellen sowie bei der kaiserlichen Regierung hohes Ansehen und noch heute preisen ihn viele Chinesen als einen der wichtigsten Kulturvermittler zwischen Ost und West. Seine Beiträge zum Fortschritt der chinesischen Gesellschaft, zur Verbreitung westlicher Bildungskonzepte und besonders zur Vermittlung der chinesischen Kultur und Philosophie ins Abendland sind zweifellos herausragend und entsprechen durchaus den Maßstäben, die man in China an heroische Persönlichkeiten anlegt.

\section{Ein gerechter und warmberziger Missionar}

Richard Wilhelm (1873-1930), der als junger Missionar des Allgemein-EvangelischProtestantischen Missionsvereins nach China gegangen war, kehrte nach 25 Jahren Aufenthalt in China als Konfuzianer nach Europa zurück. Während seines Aufenthaltes kam er durch seine vielfaltige Tätigkeit mit weiten Kreisen der chinesischen Bevölkerung in Berührung und vertiefte sich wie selten ein Europäer in die Verhältnisse und das Geistesleben Chinas. Je mehr er sich mit der chinesischen 
Welt beschäftigte, desto größere Sympathie und höhere Verehrung brachte er dem ältesten Kulturkreis der Erde und dessen geistigen Blüten entgegen, so dass er seine missionarische Aufgabe zu hinterfragen begann. Schließlich sah er seine Mission vielmehr darin, als geistiger Mittler zwischen China und Europa zu fungieren und so seinen Beitrag zur Verständigung zwischen Ost und West zu leisten.

Im Mai 1900 kam Richard Wilhelm nach der deutschen Besetzung der Jiaozhou-Bucht nach Qingdao. Vom Anfang an trat er als ein gerechter und warmherziger Missionar unter den Chinesen auf. Seiner eigentlichen Aufgabe, die Chinesen zum Christentum zu bekehren, kam er kaum nach. Vielmehr lag es ihm am Herzen, die Kultur des Landes und die Menschen möglichst schnell kennenzulernen und zu verstehen. Bereits kurz nach seiner Ankunft lernte er auf Ratschlag seines Vorgängers Ernst Faber Chinesisch und machte rasche Fortschritte. Je mehr er auf die Menschen einging, desto mehr Respekt und Sympathie entwickelte er. „Ich habe das große Glück gehabt, fünfundzwanzig Jahre meines Lebens in China zu verbringen. Ich habe Land und Volk lieben gelernt wie jeder, der lange dort weilte." ${ }^{4}$ Er erinnerte sich später an diese Erfahrung und beschreibt, wie er als Missionar keinen Chinesen bekehrt oder getauft hat, sondern selbst vielmehr von der chinesischen Kultur bekehrt und schließlich zum Konfuzianer wurde.

Doch teilten bei weitem nicht alle Europäer, die in dieser Zeit nach China kamen, seine Lernbereitschaft und Offenheit der unbekannten Kultur gegenüber, viele zeigten sich ignorant und überheblich, die westlichen Großmächte kamen nicht, um zu lernen, sondern um zu herrschen:

Wenn es zu Streitigkeiten gekommen war, waren Kanonenboote erschienen und hatten Brandschatzungen eingetrieben, und immer mehr wurde das Volk von den Fremden und ihren Anhängern bedrückt. War nicht erst vor kurzem die Jiaozhou-Bucht weggenommen worden, angeblich, weil einige Missionare von Räubern ermordet worden waren? Und hatten nicht darauf die anderen europäischen Mächte, statt den Raub zu verhindern, dieses Beispiel nachgeahmt? Wurde nicht immer wieder davon gesprochen, dass man China aufteilen wollte wie eine Melone?5

Diese Passage zeigt uns, dass Wilhelm sich kurz nach seiner Ankunft bereits Gedanken über die eigentlichen Ziele der brutalen militärischen Invasion der westlichen Mächte in China machte. Er meinte, das chinesische Volk könnte durchaus als das freundlichste, ehrlichste und liebenswürdigste der Erde gelten, wenn es vom Westen fair und respektvoll behandelt würde, was jedoch nur allzu selten geschah. China wurde durch imperialistische Mächte aus Übersee zerstückelt und aufgeteilt, das Volk unterdrückt und beraubt von Fremden aus fernen Ländern. Der Hass gegen die Invasoren wuchs.

Als der Konflikt schließlich im sog. Boxeraufstand eskalierte, wies Wilhelm mit seiner wissenschaftlichen Genauigkeit darauf hin, dass die Übersetzung „Boxer“ falsch war, denn

4 Richard Wilhelm: Die Seele Chinas, Frankfurt am Main 1980 [1928], S. 25.

5 Ebd., S. 45. 
[e]s gab auf dem Lande allenthalben Selbstschutzvereinigungen gegen das Räuberwesen, das Wege und Stege unsicher machte. Diese Selbstschutzverbände nannten sich I Ho T'uan (Vereinigungen zum Schutz der öffentlichen Ruhe). Es heißt, dass kurz nachdem in Deutschland das Wort von der gepanzerten Faust gefallen war, dieser Titel umgewandelt wurde in I Ho K'üan (Faust zum Schutz der öffentlichen Ruhe). Das wurde dann fälschlich mit dem Wort Boxer übersetzt, obwohl von Boxen bei der ganzen Sache nicht die Rede war. ${ }^{6}$

In den Konflikten zwischen den Bauern und Invasoren spielte Wilhelm stets eine vermittelnde Rolle, denn er war der Überzeugung, dass die Kirche auf keinen Fall die Gewaltanwendung befürworten dürfe, sei es auch zu angeblich guten Zwecken. Meistens gerieten die Parteien wegen mangelnder Kommunikation und Missverständnissen in Konflikt: „Ich war überzeugt, dass es sich um gegenseitige Missverständnisse handle. Und trotz Abreden bedenklicher Freunde entschloss ich mich, in die Gegend zu reisen, um zu versuchen, durch Vermittlung Menschenleben zu retten."7

Wilhelm verhandelte, der Gefahr für sein eigenes Leben nicht achtend, zwischen den Konfliktparteien, indem er sich vor allem als Dolmetscher betätigte, was letztlich erfolgreich war: Die Bauern in den Dörfern legten ihre primitiven Waffen nieder und die deutschen Truppen zogen ab. Hunderte von Leben wurden damit gerettet, vor allem Frauen und Kinder. Eine rührende Dankbarkeit der Bevölkerung war die Folge, man überreichte ihm seidene Ehrenbehänge und schließlich wurde ihm auf Antrag des Provinzialgouverneurs von der chinesischen Regierung sogar das Ehrenzeichen eines Mandarins für seine Heldentaten verliehen.

Gegenüber dem ,Boxeraufstand' zeigte Wilhelm eine völlig andere Haltung als die deutsche Besatzungsbehörde in Qingdao. Obwohl er strikt gegen den Missbrauch der Gewalt durch die Boxer war, sprach er sich dafür aus, anstatt Gewalt mit Gewalt zu beantworten und die christliche Religion den Chinesen aufzuzwingen vielmehr den Ursachen der Boxer-Rebellion auf den Grund zu gehen und die Furcht und Besorgnisse der Chinesen zu erkennen und ernst zu nehmen. Doch fanden seine Worte kein Gehör. Als Missionar sah Wilhelm seine einzige Möglichkeit darin, sich auf das einfache Leben nach christlichen Grundsätzen zu beschränken, in Schule und Hospital zu wirken, mit den Menschen zusammenzuleben und ihnen innerlich nahezukommen. Er meinte, eine Kirche könne sich nur von selbst konstituieren, sie könne nicht unter der Leitung von Fremden - oft solchen von niedriger gesellschaftlicher Bildung und ohne Taktgefühl - stehen, ohne selbst zu moralischer Inferiorität verdammt zu sein. ${ }^{8}$

Durch seine hartnäckige volksnahe Arbeit gewann Wilhelm allmählich die Freundschaft und das Vertrauen der Bevölkerung. Auf seinen Vorschlag wurde in der Gemeinde Gaomi eine moderne Grundschule geründet. Die lokale Behörde

\footnotetext{
Ebd., S. 45.

Ebd., S. 53.

Ebd., S. 55.
} 
verlieh Wilhelm den Titel des Schulrats als Zeichen ihrer Dankbarkeit und Anerkennung seiner Bemühungen. Ein solche innige und freundschaftliche Beziehung zur chinesischen Bevölkerung war für christliche Missionare dieser Zeit keineswegs selbstverständlich, sie bildete den Grundstein der späteren Verehrung Wilhelms in China.

\section{Ein engagierter und erfolgreicher Pädagoge}

Nach der Eroberung der Bucht von Jiaozhou war Deutschland bestrebt, seine koloniale Macht in China langfristig zu etablieren. Qingdao sollte dabei zum Bollwerk für die Verbreitung und Förderung deutscher Kultur in Ostasien ausgebaut werden und als Sprungbett ins chinesische Binnenland dienen. Zu diesem Zweck unterstützte die deutsche Kolonialinstanz nachdrücklich die Mission unter dem Volk und die Einführung des modernen, westlichen Bildungswesens in China.

Richard Wilhelm wusste diese Chance zu nutzen. 1898 gründete die deutsche Besatzungsbehörde eine Bildungskommission und richtete 26 Schulen nach neuestem Modell ein. Ein Jahr danach stellte Wilhelm einen Antrag auf Errichtung einer pädagogischen Schule, in der die künftigen Lehrer für die modernen Schulen ausgebildet werden sollten. Der Antrag kam der Kolonialregierung gelegen und wurde gleich genehmigt. So entstand die erste deutsche Schule in China unter Führung von Richard Wilhelm. Im Juni 1901 nahm die Deutsche Schule (höhere Knabenschule des Allgemeinen Evangelisch-Protestantischen Missionsvereins), finanziell von der Weimarer Mission unterstützt, den Betrieb auf. Dreißig Schüler wurden von drei europäischen Lehrern und fünf chinesischen Gelehrten unterrichtet. Wilhelm selbst leitete die Schule, besorgte moderne Laborinstrumente aus Deutschland und erarbeitete persönlich ein umfangreiches Curriculum mit den Fächern Chinesisch, Deutsch, Physik, Chemie, Mathematik, Geometrie, Buchhaltung u. a. für die Schüler. Insbesondere das Fach Mathematik löste in Qingdao lebhafte Diskussionen aus, denn die Chinesen waren neugierig und betrachteten die Einführung von Mathematik ins Schulcurriculum als bahnbrechende Maßnahme. Bis 1914 hatte die Deutsche Schule Bestand, dann musste Deutschland Qingdao an die Japaner abtreten. Seither erlebte die Schule ein wechselhaftes Schicksal, wurde nacheinander von der Basler Mission, der lokalen Handelskammer und schließlich der chinesischen Regierung übernommen.

Die Schule bot ein siebenjähriges Bildungsprogramm an, drei Jahre in der Unterstufe und vier Jahre in der Oberstufe. Die Schüler mussten bei der Aufnahmeprüfung einen chinesischen Sprachtest bestehen und dann in der Schule Deutsch lernen, denn viele Kurse aus dem Bereich der Naturwissenschaften wurden auf Deutsch gehalten. Wilhelm legte großen Wert auf die Qualität der Lehre und engagierte nur hochrangige Gelehrte. Er setzte sich für die Chancengleichheit bei der Volksbildung ein und achtete darauf, dass die chinesische Kultur als Grundlage, die westliche Wissenschaft als Aufbau in der Lehre fungierten. Dieser Bildungsansatz 
galt damals als äußerst modern und machte rasch Schule, eine neue Ära des Bildungswesens hatte begonnen. In der Lehre achtete Wilhelm auf die Anwendung moderner, didaktischer ausgefeilter Unterrichtsmethoden, um den Denkhorizont der Schüler zu erweitern. Schon bald erlangte die Schule auch überregionale Bekanntheit. Der chinesische Gouverneur der Provinz Shandong, Zhou Fu, besuchte die Schule und war so begeistert, dass er ihr sogleich das Recht erteilte, Schüler zur Prüfung an die Shandong Universität zu schicken. Tatsächlich bestanden in den folgenden Jahren mehrere Schüler die Aufnahmeprüfung zum Hochschulstudium, ein erster großer Erfolg der modernen Bildungsansätze in China. Besonders auch das einfache Volk profitierte von der neuen Schule, da nun erstmals auch Kinder aus ärmeren Familien die Möglichkeit erhielten, eine Schule zu besuchen und mit der Bildung die Basis für gesellschaftlichen Aufstieg zu erlangen. Mehr als tausend Absolventen sollten in den kommenden Jahren aus der Schule hervorgehen und zahlreiche neue Einrichtungen nach dem Vorbild von Wilhelms Schule wurden in der Region gegründet, für die Lehrkräfte mit modernem Bildungsbewusstsein und didaktischer Kompetenz ausgebildet wurden.

Der Erfolg von Wilhelms Schule in Qingdao hatte die Aufmerksamkeit der Qing-Regierung auf sich gezogen und ihm Anerkennung und Belohnung eingetragen. Richard Wilhelm wurde vom Kaiser der Titel eines kaiserlichen Hochbeamten vierten Ranges verliehen samt der prächtigen Beamtentracht, die er künftig bei Feierlichkeiten und auf Abschiedsfotos mit seinen Zöglingen zu tragen pflegte.

1905 wurde Wilhelm von der Basler Mission beauftragt, eine Mädchenschule zu gründen, die nach dem Namen seiner Ehefrau Salome benannt wurde. Die Schule wurde zum Vorreiter der modernen Mädchenschulen in der Provinz Shandong und bot in Übereinstimmung mit dem deutschen Mädchenschulkonzept Kurse wie Deutsch, Chinesische Literatur, Mathematik, Geographie, Naturkunde, Zeichnen, Musik, Nähen und Sticken an. Das Bildungsprogramm bestand aus drei Jahren Unterstufe und sechs Jahren Oberstufe. Zum Zeitpunkt der Gründung der Schule gab es nur fünf Schülerinnen, 1907 schon 28 und schließlich 62 im Jahr 1908. Die rasch steigende Zahl der Schülerinnen zeigt den Erfolg des modernen, westlichen Bildungswesens und deren Anerkennung von Seiten der Chinesen.

Zur selben Zeit gründete Richard Wilhelm eine andere wichtige Institution, die Konfuzius-Gesellschaft, der eine Bibliothek mit 3000 Büchern angegliedert war. Sie avancierte alsbald zu einem beliebten Treffpunkt der chinesischen Gelehrten, die vor dem Krieg flüchteten und in Qingdao Schutz suchten, woraus sich schnell ein intensiver Austausch Wilhelms mit ihnen entwickelte.

Auch im Bereich des Gesundheitswesens war der deutsche Missionar aktiv. Er gründete in der Stadt Qingdao zwei Krankenhäuser, auf dem Land zwei Krankenstationen und beteiligte sich persönlich neben dem Schuldienst an der ambulanten Behandlung, was seine Beliebtheit bei der Bevölkerung noch steigerte. Wilhelms Beitrag im Bereich der Bildung und Gesundheit ging weit über seine eigentliche Missionsarbeit hinaus, so dass manche Deutsche ihn verspotteten, dass er anstatt 
einer kirchlichen Mission eine Kulturmission in China gestartet habe, worin sich zugleich die Besorgnis um seine missionarische Verpflichtung widerspiegelt.

Als Pädagoge pflegte Richard Wilhelm auch noch nach seiner Rückkehr nach Deutschland einen regen Kontakt mit den chinesischen Studenten vor Ort, führte sie in die deutsche Philosophie und Literatur ein und übersetzte kurze Artikel von Kant und Goethe ins Chinesische. Er engagierte sich sehr dafür, den Chinesen die Gedanken und künstlerischen Leistungen berühmter deutscher Philosophen und Dichter nahezubringen. Selbst nach seiner Rückkehr nach Deutschland vergaß Wilhelm seine seelische Heimat China aber nie und lud wiederholt chinesische Gelehrte ein, Vorlesungen an seinem Chinainstitut zu halten, so zum Beispiel Hu Shi, einen anerkannten Denker und Literaten Chinas, Vertreter der Neuen-KulturBewegung und Rektor der Peking Universität.

Die beiden Söhne Wilhelms sind später in die Fußstapfen ihres Vaters getreten und Professoren an der Universität Peking geworden, stets bemüht, die Lebensarbeit ihres Vaters fortzusetzen. Einer von ihnen ist für seine sinologischen Arbeiten bekannt und noch heute sind die deutschen Sinologen stolz darauf, sich Schüler von Richard Wilhelm nennen zu dürfen. Um seine Leistung auf dem Gebiet der Kulturvermittlung und Völkerverständigung zu würdigen, hat die Bundesrepublik Deutschland den Richard-Wilhelm-Preis für Literaturübersetzungen eingerichtet.

\section{Sinologe und Kulturvermittler zwischen Ost und West}

Als Wilhelm im Jahre 1900 in Qingdao ankam, trat er in ein völlig neues Umfeld mit einer ganz eigenen kulturellen Tradition: Sprache, Schrift, Bräuche und Gewohnheiten mussten ihm zunächst fremd und unverständlich scheinen, was ihn nicht nur daran hinderte, seine Missionsarbeit zu leisten, sondern auch Kontakte zu Einheimischen zu knüpfen. Sein Vorgänger, der Missionar Ernst Faber, legte ihm ans Herz, sich die chinesische Sprache und Schrift schnellstmöglich anzueignen, wenn er die Kultur und Menschen Chinas verstehen und dadurch die Missionsarbeit zügig vorantreiben wolle, und tatsächlich war Wilhelm fest entschlossen, sich den Zugang zu dieser fremden Kultur zu erarbeiten. Mit großem Interesse und aufrichtiger Bewunderung erklomm er die Chinesische Mauer, die Berge Lao und Tai, befuhr den Jangtse und den Gelben Fluss, bereiste die Millionenstädte wie Peking, Datong, Shanghai, Suzhou und Hangzhou. Auf diesen Studienreisen widmete er sich ganz dem Land und seinen Bewohnern, studierte zugleich unermüdlich die chinesische Sprache und Schrift.

Wilhelm war zunehmend beeindruckt von dem „heiteren China“ und von der „großen Harmonie des Lebens“. ${ }^{9}$ Er entschied sich, den Ursprung dieses harmonischen Geistes zu erforschen und die Geheimnisse der chinesischen Zivilisation in deren heiligen Schriften und philosophischen Lehren zu erkunden. Schnell

$9 \quad$ Ebd., S. 307. 
hatte er sich so profunde Sprachkenntnisse angeeignet, dass er die Klassiker der chinesischen Philosophie und Literatur lesen konnte und sich mit renommierten Großgelehrten wie Kang Youwei (Philosoph, Politiker und Reformer der SpätQing-Dynastie ) und Lao Naixuan (Großgelehrter, entschlossener Verteidiger des feudalistischen Altchinas, bewandert in der traditionellen chinesischen Philosophie) darüber austauschte. Zu seiner Lektüre zählten etwa das Tao Te King, die Schriften von Lao Tse, die Gespräche von Konfuzius, die Wabren Worte vom südlichen Blütenland, die Schriften von Dschuang Dsi, die Lehre vom Mittelmaß. Je besser er mit diesen Schriften vertraut wurde, desto fataler schien es ihm, dass diese wertvollen Texte noch nie ins Deutsche übersetzt worden waren und seine Landsleute deshalb keinerlei oder nur eine sehr oberflächliche Vorstellung von der Kultur, Geschichte und Philosophie Chinas haben konnten. So entschloss er sich, diese alten chinesischen Meisterwerke ins Deutsche zu übersetzen. Zugleich schrieb er zahlreiche Zeitungsartikel und kleinere Texte, die die chinesische Kultur vorstellen, erklären und die Deutschen mit ihr vertraut machen sollten.

Während seiner Jahre in China und nach der Rückkehr nach Deutschland übersetzte Wilhelm daher verschiedenste chinesische Klassiker ins Deutsche und veröffentlichte sie als Hauptbestandteil seiner China-Reihe, eine Arbeit, die bis heute nachwirkt. Zudem verfasste er eigene Bücher über die chinesische Kultur wie z. B. Chinesische Lebensweisheit, Die Seele Chinas, Religion und Philosophie Chinas, Chinesische Wirtschaftspsychologie, Lao Tse und Taoismus, Kung-Tse: Leben und Werk. Innerhalb der chinesischen Philosophie widmete Wilhelm dem Studium des Konfuzianismus die größte Aufmerksamkeit. Hielt er Kant gleichsam für einen westlichen Heiligen, so war Konfuzius für ihn ein östlicher. Dessen Hingabe an die Bildung, die soziale Ordnung, die Harmonie der zwischenmenschlichen und gesellschaftlichen Beziehungen und sein beharrlicher Wille und Opfersinn ließen ihn für Wilhelm zur größten Persönlichkeit der chinesischen Geschichte werden. Obwohl Wilhelm sein eigenes Leben zwar nicht im Sinne des Konfuzianismus gestaltete, so hinderte ihn das nicht daran, für dessen Ideal zu plädieren. Er betrachtete Konfuzius als einen Wächter des alten chinesischen Geistes und glaubte, dass sich seine Lehren auf die Entwicklung Chinas - und auch Deutschlands - positiv auswirken könnten, wenn sie nur gehört und verstanden würden.

Unter den zeitgenössischen chinesischen Philosophen bewunderte Wilhelm Kang Youwei am meisten und stand ihm am nächsten, der in seinen späteren Jahren in Qingdao lebte. Aufgrund ihrer gemeinsamen akademischen Interessen lernten sie einander kennen und respektieren. Wilhelm hielt Kang Youwei für einen neuen Heiligen, gleichsam einen zweiten Konfuzius. In seinem Buch Die Seele Chinas warf Wilhelm einen dokumentarischen Rückblick auf die chinesische Geschichte und Kultur, worin er auch die drei Hauptwerke Kangs, die Prüfung der konfuzianischen Klassiker, Prüfung der Konfuzius-Reformation und das Datong-Buch, bespricht und kommentiert. Er war hoch begeistert von Kangs Vorstellungen über gesellschaftliche Reformen und beteuerte, dass Kangs Gedanken und Taten 
ein unerbittlicher Schock für das tausende von Jahren unveränderte feudale Gesellschaftssystem Chinas und die stagnierende Qing-Dynastie seien. Für Wilhelm war Kang Youwei der revolutionärste Akademiker seiner Zeit. Obwohl Kang großen Respekt vor Konfuzius hatte, standen viele seiner Vorstellungen im Gegensatz zu traditionellen Ansichten und Konventionen und sorgten dafür, dass diese auch von anderen zunehmend kritisch hinterfragt wurden.

In der Seele Chinas stellte Wilhelm auch Reformer wie Zhang Taiyan, Liang Qichao und den Revolutionär und Gründer der Republik China, Sun Yat-sen, vor und unterstützt ihre Beiträge zum Versuch, Chinas Rückständigkeit zu bekämpfen. Deutlich bringt er seinen Respekt vor der chinesischen Kultur und seine Zuversicht in deren Neugeburt zum Ausdruck:

Heute entwickelt sich das Leben in China in fieberhafter Eile. Jeder Tag bringt neue Ereignisse und Entwicklungen, und hinter den lauten Tagesereignissen und Kämpfen vollzieht sich etwas ganz Großes: das Auftauchen einer neuen Welt... Ich habe das große Glück gehabt, fünfundzwanzig Jahre meines Lebens in China zu verbringen. Ich habe Land und Volk lieben gelernt wie jeder, der lange dort weilte. Aber gerade die jetzt vergangenen fünfundzwanzig Jahre waren besonders wichtig, weil sie es waren, in denen Altes und Neues sich trafen. Ich habe noch das Alte China gesehen, das für die Jahrtausende zu dauern schien. Ich habe seinen Zusammenbruch miterlebt und habe erlebt, wie aus den Trümmern neues Leben blühte. Im Alten wie im Neuen war doch etwas Verwandtes: eben die Seele Chinas, die sich entwickelte, aber die ihre Milde und Ruhe nicht verloren hat und hoffentlich nie verlieren wird. ${ }^{10}$

Nach der Revolution von 1911 und der darauffolgenden Abdankung des letzten Kaisers der Qing-Dynastie flüchteten eine ganze Reihe königlicher Verwandter, Minister, Hofbeamter und renommierter Gelehrter der gestürzten Dynastie nach Qingdao, wo sie den Schutz der deutschen Kolonialmacht suchten. In dieser Zeit lernte Wilhelm viele gebildete und einflussreiche Chinesen kennen und stand mit ihnen in lebhaftem geistigem Austausch. Unter ihnen waren auch der VizeBildungsminister Lao Naixuan, renommierter Altkonfuzianer, der Wilhelm bei der Übersetzung vom Buch der Wandlungen beratend beistand, sowie Liu Yanchen, Rektor der Pädagogischen Hochschule Peking; Zhou Fu, der Ex-Gouverneur der Shandong Provinz und Prinz Fu Wei, dessen Großvater die Verhandlung zwischen China und den westlichen Alliierten nach der Niederlage Chinas im Zweiten Opiumkrieg geleitet hatte. Die von Wilhelm gegründete Konfuzius-Gesellschaft war damals ein beliebter Treffpunkt dieser Gruppe. Wilhelms Verständnis der chinesischen Kultur wurde zweifelsohne stark von ihnen beeinflusst, doch hinderten sein Respekt vor Konfuzius und seine Vorliebe für die Tradition und Philosophie Chinas ihn nicht daran, auch Kontakte zu den Vertretern der Neuen-KulturBewegung zu pflegen, die China von der alten, überkommenen Tradition lösen wollten und sich für die Demokratie, moderne westliche Wissenschaft und die Öffnung und Reform Chinas einsetzten. Zu dieser Gruppe gehörten Cai Yuanpei,

10 Ebd., S. 25. 
der Rektor der Universität Peking, an der die Studentenbewegung des Vierten Mai ausbrach, Chen Duxiu, Gründer der kommunistischen Partei Chinas, Liang Shumin, angesehener Pädagoge, Denker und Repräsentant der Bewegung des Vierten Mai sowie Yan Fu, Rektor der Universität Fudan, Aufklärer, Denker und Reformer. Auch zu ihnen knüpfte Wilhelm freundschaftliche Kontakte und viele von ihnen lud er später nach Deutschland zu Vorträgen ein, nachdem er den Lehrstuhl für Sinologie am China-Institut in Frankfurt übernommen hatte. Als Sinologe genoss Wilhelm wegen seiner ausgezeichneten Übersetzung der chinesischen Klassiker und seiner hervorragenden Leistung in der Kulturvermittlung sowie seines persönlichen Engagements für die chinesischen Gelehrten und Studenten eine hohe Anerkennung in der chinesischen Bevölkerung und Regierung.

Von seinen 57 Lebensjahren verbrachte Richard Wilhelm fast die Hälfte in einem fernen, fremden Land. Er kam als Missionar nach China und widmete seine ganze Energie der Einführung des modernen Bildungssystems und der Verbesserung der Gesundheit der Bevölkerung. Mit außerordentlichem Fleiß und unermüdlichem Engagement vertiefte er sich in die chinesische Kultur und wurde zu einem ihrer tiefsten Verehrer. Nach seiner Rückkehr nach Deutschland setzte er seine Vorhaben fort, indem er all seine Bemühungen der Ausbildung von Sinologen in Deutschland und der Vermittlung chinesischer Kultur in Deutschland widmete. Auch nach der Gründung der Volksrepublik China wurde Wilhelm wegen dieses jahrelangen Einsatzes von der akademischen Gemeinschaft in China gelobt und anerkannt, in mehreren Dokumentationen über den kulturellen Austausch zwischen China und dem Westen würdigte man seine Arbeit. Für die Chinesen wurde er zu einer heroischen Figur, zu einer Personifikation von kultureller und geistiger Offenheit, von einer vorurteilsfreien Betrachtung und Wertschätzung der Eigenheiten und spezifischen Qualitäten einer scheinbar fremden Kultur. Von dieser Wertschätzung, die Richard Wilhelm noch heute als Kulturheros in China genießt, zeugt das Denkmal in Qingdao, das, geschaffen von dem Künstler Diao Yunbo, dem Vizevorsitzenden des Qingdao-Bildhauerverbandes, im Dezember 2009 zum 110-jährigen Jubiläum der Wilhelm Schule eingeweiht wurde (Abb. 1). Die Bronzebüste steht auf einem Postament, das Wilhelms Lebensleistung in chinesischer und englischer Sprache würdigt.

\section{Einfluss auf Hermann Hesse}

Richard Wilhelm hat sich durch seine Übersetzungen der chinesischen Klassiker im Westen einen großen Namen gemacht und das europäische Denken zu Beginn des 20. Jahrhundert stark beeinflusst. Durch seine exakte Übersetzungsarbeit und allgemeinverständlichen Erklärungen hat Wilhelm die chinesische Philosophie mit ihren Lebensweisheiten vielen Menschen im Westen zugänglich gemacht, in den Jahren während und nach den beiden Weltkriegen dienten sie vielen Notleidenden als geistige Oase und seelischer Trost. Bei zahlreichen 


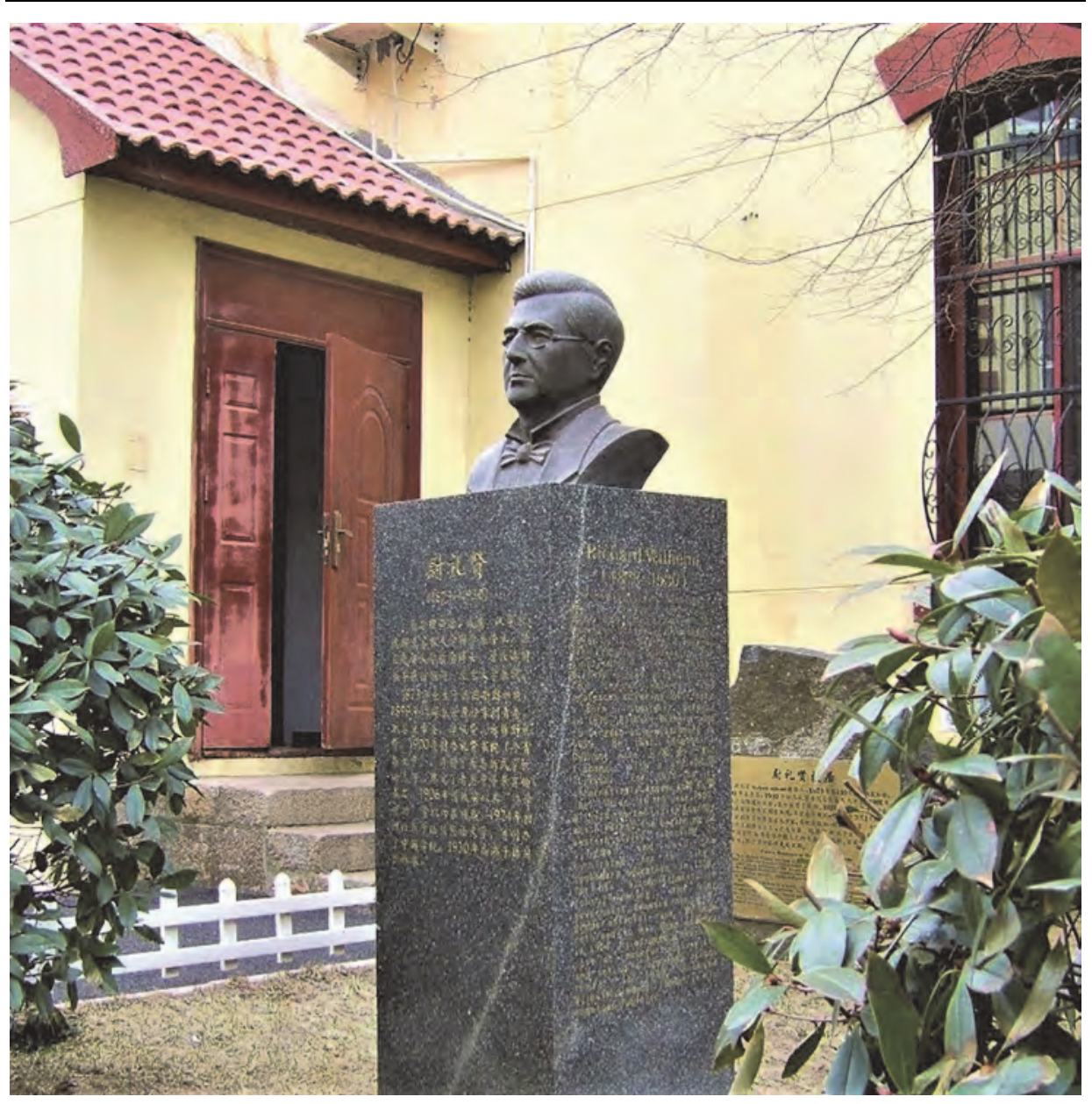

Abb. 1: Bronzebüste für Richard Wilhelm vor der Mittelschule in Quingdao, Jiangsu Lu 8-10, früher Kaiserliche Gouvernements-Schule.

Schriftstellern und Dichtern haben seine Übersetzungen Spuren hinterlassen. Als ein prominentes Beispiel soll hier nur Hermann Hesse genannt werden, der die Übersetzungen Wilhelms intensiv studierte und auch persönlichen Austausch mit ihm pflegte.

Wiederholt hat Hesse in Briefen und Aufsätzen darauf hingewiesen, dass sich ihm die chinesische Gedankenwelt erst durch Richard Wilhelm richtig erschlossen habe. Obwohl seine Studien weit über die klassische chinesische Philosophie hinausgingen, bildeten die Wilhelmschen Übersetzungen den Ausgangspunkt seiner intellektuellen Auseinandersetzung mit der chinesischen Geisteswelt. In einem Brief an Wilhelm vom 4. Juni 1926 schrieb Hesse:

Sie sind mir seit langem lieb und wichtig. Ich verdanke Ihnen so ziemlich alles, was ich an Beziehungen zum Chinesischen habe, das mir, nach einer vieljährigen mehr indi- 
schen Orientierung, sehr wichtig wurde [...]. Desto dankbarer bin ich für die stillen geistigen Liebesbeziehungen, die mir das Leben trotzdem gebracht hat, und zu ihnen gehört das China, das ich durch Sie kennlernte, und damit die Dankbarkeit gegen Sie und Ihr Werk. Sie einmal aussprechen zu dürfen, ist mir eine Freude. ${ }^{11}$

Der Brief zeigt, wie sehr Hesse Richard Wilhelm und dessen Werk wertschätzte. Durch seine hartnäckigen Bemühungen wurde eine ganze Reihe chinesischer Klassiker den deutschen Lesern zugänglich gemacht. 1909 erschien sein erstes Buch Kung Futse. Gespräche, in den Jahren darauf kamen Laotse. Tao Te King. Das Leben des Alten vom Sinn und Leben (1910), Liä Dsi. Das wahre Buch vom quellenden Urgrund (1911), Dschuang Dsi. Das wahre Buch vom südlichen Blütenland (1912), I Ging. Das Buch der Wandlungen (1924), Frübling und Herbst des Lü Bu We (1928) und noch zahlreiche Vorträge und Aufsätze über die Kultur und das Alltagsleben Chinas zustande. Diese Arbeiten brachten ihm nicht nur den Ruhm des größten deutschen Sinologen dieses Jahrhunderts ein und erregten eine Welle sogenannter China-Mode in Deutschland, sondern haben auch "die durch alle Jahrtausende lebendige geistige Wurzel des chinesischen Geistes mitgebracht und in den Boden Europas gepflanzt $^{\text {"12 }}$ und dadurch das Weltbild der Europäer wesentlich verändert.

Hesses positives Bild von China, dem Land der Weisheit und der Lebensharmonie, ist zum größten Teil Richard Wilhelms Übersetzungen zu verdanken, die seine wesentliche Quelle darstellten und wurden die Ursache seiner Liebe zur chinesischen Philosophie und Kultur. Von 1910 bis in die dreißiger Jahre hinein las Hesse fast alle Wilhelmschen Übersetzungen der chinesischen Klassiker und machte wiederholt durch Rezensionen in den Zeitungen und Zeitschriften auf Wilhelm und seine Arbeiten aufmerksam. Die gemeinsame Liebe der beiden Männer zur chinesischen Philosophie trug auch zu ihrer persönlichen Freundschaft bei. Leider ist diese nur schlecht dokumentiert, sodass sich davon nur ein unvollständiges Bild gewinnen lässt. Doch Hesse muss Wilhelm bereits vor dem Lesen der Gespräche von Konfuzius (1909) gekannt haben. Denn wie Frau Wilhelm in der Biographie ihres Mannes angibt, habe sich Richard Wilhelm, der damals noch junger Vikar war, bei einem Hochzeitsfest mit Maria Bernoulli befreundet, die später Hesses Frau werden sollte. Mit ihr korrespondierte Wilhelm auch noch aus China. Der persönliche Kontakt zwischen beiden entstand allerdings erst, als Hesse durch Wilhelms Übersetzungen in ihm einen Gleichgesinnten und Verehrer des ostasiatischen Geistes erkannte. Es entwickelte sich ein langjähriger Briefwechsel, wobei China und seine Weisheiten stets im Mittelpunkt standen. Nur zwei persönliche Begegnungen lassen sich quellenmäßig sicher nachweisen, es werden aber vermutlich mehrere Treffen stattgefunden haben. Einmal begegneten sich die beiden im Herbst 1926 bei ihrem gemeinsamen Freund und China-Verehrer Georg Reinhart

11 Hermann Hesse: Die Briefe, Bd: 4: „Ich bin ein Mensch des Werdens und der Wandlungen“, 1924-1932, hg. von Volker Michels, Berlin 2016, S. 149-150.

12

Carl Gustav Jung: Zum Gedächtnis Richard Wilhelms, in: Das Geheimnis der Goldenen Blüte. Ein chinesisches Lehrbuch, übersetzt und erläutert von R. Wilhelm mit einem europäischen Kommentar von C. G. Jung, Zürich 1929, S. XVII. 
in Zürich und ein anderes Mal im Dezember desselben Jahres in Frankfurt, als Hesse seine Werke an der Universität Frankfurt vorstellte. Bedauerlicherweise blühte ihre Freundschaft nur auf geistiger Ebene, da die beiden grundverschiedene Charaktere waren. Während Hesse zu Ruhe, Einsamkeit und Melancholie neigte, war Wilhelm ein heiterer und geselliger Mensch voller Lebensenergie. Diese charakterlichen Unterschiede, die trotz allem dem geistigen Austausch der beiden nicht im Wege standen, beschreibt Wilhelm selbst in einem Antwortschreiben vom 8. Juni 1926:

\section{Lieber Herr Hesse,}

Vielen Dank für Ihren Brief vom 4. Juni, der mich sehr gefreut hat. Es geht mir mit diesen Beziehungen ganz ähnlich wie Ihnen. Ich freue mich, wenn ich ihnen im Leben irgendwo begegne, sei es in Ihren Büchern oder bei Freunden wie Georg Reinhart oder Gundert. Und diese mannigfachen Beziehungen spinnen sich weiter auch ohne dass man einander schreibt; denn auch mit dem Briefschreiben geht es mir ganz wie Ihnen. Aber ich möchte Sie einmal persönlich sehen, einen Abend mit Ihnen zusammen sein und - wenn Sie nicht Antialkoholiker sein sollten - einen guten Wein mit ihnen trinken und über vieles zusammen reden oder schweigen.

Lassen Sie sich durch meine Verbindung mit Keyserling nicht irre machen. Und auch nicht durch mein ,Weltwesen'. Wenn man die Welt so durchschaut hat wie Sie und ich, so kann man sich auf doppelte Art verbergen: in der Einsamkeit, wie Sie und in der Welt, wie ich. Aber ich glaube wir werden uns verstehen, und ich kann Ihnen aus dem alten China mehrere Herren vorstellen wie Tschuangtse u.a., die gänzlich unsozial waren. Meister Kung hat viel unter ihnen zu leiden gehabt und verstand sie nur zu gut. (Besser als er sich seinen Jüngern gegenüber den Anschein gab).

Viele herzliche Grüße

Ihr Richard Wilhelm ${ }^{13}$

1930 starb Richard Wilhelm. Bei seinem Tod schrieb Hesse in der Berliner Zeitschrift Bücherwurm:

Langsam wächst der Kreis derer, welche gemerkt haben, dass Wilhelms Lebenswerk zu den paar großen unserer Zeit gehört [...]. Inmitten dauernder Missverständnisse stand er lächelnd, freundlich, chinesisch-weise und tat in Ruhe sein großes Werk, dessen Umfang und Bedeutung von der öffentlichen Meinung Deutschlands noch gar nicht begriffen worden ist. Er hat Zeit, er hat nicht bloß für eine Generation gearbeitet. ${ }^{14}[\ldots]$. Es ist bei uns in den letzten Jahrzehnten sehr viel entdeckt, übersetzt und neu herausgegeben worden. Nichts von alledem ist mir im Laufe von beinahe 20 Jahren wichtiger und teurer geworden als Wilhelms deutsche Ausgabe der chinesischen Klassiker, sie haben mir und vielen eine Welt erschlossen, ohne die wir nicht mehr leben möchten. ${ }^{15}$

Im Jahre 1956 erschien schließlich die von Wilhelms Frau Salome verfasste Biographie Richard Wilhelm. Der geistige Mittler zwischen China und Europa. Gleich nach

13 Zitiert nach Adrian Hsia: Hermann Hesse und China, Frankfurt am Main 1974, S. 325.

14 Hermann Hesse: Sämtliche Werke, Bd. 19: Die Welt im Buch. IV: Rezensionen und Aufsätze aus den Jahren 1926-1934, hg. von Volker Michels, Frankfurt am Main 2003, S. 178.

15 Ebd., S. 158. 
der Veröffentlichung des Buches rezensierte Hesse es in der Weltwoche (Zürich, 27. April 1956), um seine Verehrung und sein Gedenken an diesen großen DeutschChinesen nochmals zum Ausdruck zu bringen:

Die Biographie Richard Wilhelms ist ein Buch, das keiner sich entgehen lassen sollte, der irgend Beziehungen zur Geisteswelt des Fernen Ostens hat. Darüber hinaus ist es die Lebensbeschreibung eines ganz ungewöhnlichen Zeitgenossen, eines genialen Mannes von merkwürdiger Begabung und Begnadung, von dessen Person, Leben und Werk Wirkungen ausgegangen sind, die in ihrer vollen Kraft und Vielfältigkeit erst eine kommende Generation wird erkennen können. Allgemein bekannt ist R. Wilhelm (1873 bis 1930) durch die Reihe seiner Übersetzungen und Erklärungen klassischer chinesischer Werke, von denen er einige, wie ,Lü Bu We', das ,I Ging ${ }^{\varsigma}$ und andere, als erster verdeutscht hat. Auch in meinem Leben und Denken haben diese Wilhelmschen Übertragungen und Deutungen eine wichtige Rolle gespielt, und wie mir, so hat er vielen von meiner und der folgenden Generation ein Tor auf getan und eine Botschaft übermittelt, die in unserem Leben Epoche gemacht hat.

Das Buch beginnt mit einem Nachruf, einem congenial gezeichneten Charakterbild des großen Deutsch-Chinesen aus der Feder eines anderen Meisters, Walter F. Otto, der dem andern in jahrzehntelanger Freundschaft verbunden war, hat es geschrieben, es ist der schönste mir bekannte Freundesnachruf für einen bedeutenden Mann unserer Zeit. Wer diesen Nachruf liest und dazu das Bildnis Wilhelms betrachtet, das dem Buch mitgegeben wurde, hat eigentlich schon den Mann kennengelernt, alles Wesentliche ist da. Das Bildnis zeigt ein überaus liebes Gesicht, dessen Augen ebenso lächeln wie der Mund, ein sehr freundliches, konzentriertes, leise strahlendes Gesicht, und erst wenn man es eine Weile beobachtet hat, wird einem bewußt, daß dies freundliche Lächeln viel Asiatisches hat, daß es nicht nur das Wohlwollen und die Lebensfreude eines gesunden, wohltemperierten, wohlgeordneten Mannes ausdrückt, sondern auch in allen Nuancen zwischen Schelmerei und Spott spielt wie die Geschichten, Legenden und Anekdoten um die großen Weisen des alten China.

Was ich aus diesem Bildnis lese, entspricht genau dem, was ich in Wilhelms Person und Leben immer geliebt und verehrt habe. Meine Freunde und die Leser meiner Bücher wissen, wie sehr mir erst Indien, dann China zu einer geistigen Heimat oder doch Zuflucht geworden ist. Und wenn ich irgendwo auf besonders kräftige Ablehnung, auf instinktiven $\mathrm{Haß}$ oder prinzipielles Nichtverstehenwollen stoße, so gilt diese Ablehnung beinahe immer dem Einschlag von alt-asiatischem Geist, den man in meinen Erzählungen findet. Nun, diese instinktive Furcht vor dem Fremden, Nichteuropäischen in der indischen und chinesischen Lebens- und Denkart ist nach meinem Glauben dasselbe wie jeder Rassenwahn und Rassenhaß. Etwas Bekanntes, historisch und psychologisch Begreifliches, aber etwas Rückständiges, nicht mehr Lebenbringendes, etwas, das überwunden werden muß. Unterstützt wird die Rückständigkeit nicht nur durch den Fortschrittsund Technik-Enthusiasmus des Abendlandes, sondern auch durch den Anspruch des kirchlich-dogmatischen Christentums auf Alleingültigkeit. Wenn ich nun das Bild eines Zukunfteuropäers zeichnen sollte, der diese Kluft überbrückt und auf die Dauer notwendige Synthese zwischen asiatischem und abendländischem Wesen nicht nur in Gedanken, sondern auch in Tat und Leben vollzogen hat, so würde dies ideale Menschenbild genau dem Bilde Richard Wilhelms gleichen.

Er war ein Vorläufer und ein Vorbild, ein Mensch der Harmonie, der Synthese zwischen Ost und West, zwischen Sammlung und Aktivität. Er hat in China, hat im jahrelangen intimen Umgang mit altchinesischer Weisheit und im persönlich-freundschaftlichen Austausch mit der Elite chinesischer Gelehrsamkeit weder sein Christentum noch 
sein schwäbisch-thüringisch geprägtes Deutschtum, hat weder Jesus noch Plato noch Goethe verloren und vergessen, noch seine gesunde, kraftvolle, abendländische Lust am Wirken und Bilden, er ist keinem europäischen Problem davongelaufen, hat sich keinem Anruf des aktuellen Lebens entzogen, ist weder einem denkerischen noch einem ästhetischen Quietismus erlegen, sondern hat, Stufe um Stufe, die Befreundung und Verschmelzung der beiden großen alten Ideale in sich vollzogen, hat China und Europa, Yang und Tin, Denken und Tun, Wirksamkeit und Beschaulichkeit in sich zur Versöhnung gebracht. Daher der Tonfall seiner schönen, sanft belehrenden Sprache, etwa im ,I Ging', aus dem man Goethe und Kung Fu Tse zugleich heraushört, daher der Zauber, den er auf so viele Menschen hohen Ranges in West und Ost geübt hat, daher das so weise wie freundliche, so wache wie schelmische Lächeln seines Gesichtes.

[...] Wie der Tübinger Theologiestudent zum wachen Zeitgenossen, zum Europäer, dann zum intimen Freund und Kenner Chinas wurde, was er in langen Chinajahren, besonders denen von 1914 bis 1918, dort an Herrlichem und an Rauhem erfahren hat, wie er sich die Sprache, die Kenntnis der alten Literatur, der Kunst, des Landes und schließlich die Köpfe und Herzen der besten Intelligenz Chinas eroberte, immer tätig, hilfreich, zugreifend, aber nie ungeduldig, groß im Leidenkönnen und Wartenkönnen wie im entschlossenen Handeln, wie er in China für die guten Geister des Westens, in Deutschland und Europa für das edle Erbe Chinas geworben hat, wie er schließlich in der Heimat durch ein jahrzehntelanges Übermaß an aufreibender Arbeit seine Kräfte bis zum Letzten verbrauchte, ohne doch bis zum Sterben je das innere Gleichgewicht, die Geduld, die Heiterkeit des Wissenden zu verlieren, darüber lese man in dem großartigen Buch, das seine Frau ihm gewidmet hat. ${ }^{16}$

Diese Rezension ist nicht nur eine leidenschaftliche Buchempfehlung, sondern vor allem eine verehrungsvolle Huldigung an den großen Deutsch-Chinesen Richard Wilhelm, dessen Übersetzungen chinesischer Klassiker die von asiatischer Philosophie geprägte Weltanschauung Hesses und somit sein dichterisches Schaffen wesentlich mitbestimmt haben. Hierin bekannte sich Hesse zu dem, was ihn an Wilhelm faszinierte, nämlich das Erreichen der Lebensharmonie und die Vollendung der eigenen Persönlichkeit durch die Verinnerlichung fernöstlicher Philosophie und Lebensweisheit. Nach diesem Ziel strebten sowohl Hesse selbst als auch die Protagonisten seiner Werke.

\section{Abbildungsnachreise}

Abb. 1: Wechathompage der Qingdao Mittelschule Nr. 37 (einstige Wilhelm Schule), https://mp.weixin.qq.com/s/uu_dmiKqTrNkX_2-aQGCNA.

16 Hermann Hesse: Sämtliche Werke, Bd. 20: Die Welt im Buch. V: Rezensionen und Aufsätze aus den Jahren 1935-1962, hg. von Volker Michels, Frankfurt am Main 2005, S. 332-335. 